\title{
O056: The introduction of a surgical site infection prevention bundle on a nationwide scale
}

\author{
T Hopmans ${ }^{1 *}$, L Soetens ${ }^{1}$, J Wille ${ }^{1}$, P van den Broek², M Koek¹, B van Benthem \\ From 2nd International Conference on Prevention and Infection Control (ICPIC 2013) \\ Geneva, Switzerland. 25-28 June 2013
}

\section{Introduction}

In 2009 a patient safety program, consisting of a 4-item surgical site infection (SSI)-prevention-bundle, was introduced in the Netherlands. The infection prevention bundle consist of: timely antibiotic prophylaxis, no preoperative surgical site hair removal, perioperative normothermia and hygiene discipline on the OR.

\section{Objectives}

The aim of the SSI-bundle introduced by the safety program is to reduce the SSI-rate through creating more awareness about the importance of patient safety among hospital employees. The objective is a $90 \%$ compliance with the complete SSI-prevention bundle.

\section{Methods}

Data collection (2009-2012) was incorporated in the PREZIES surveillance network for healthcare associated infections. Compliance with the four interventions was registered separately and combined in patients, who underwent a surgical procedure present on the list of indicator procedures (a selection of 13 procedures of 6 different specialties). Log binomial regression analysis was used to calculate relative risks (chance) on compliance, stratified by medical specialty, calendar time and participation period.

\section{Results}

Registration of the complete bundle was around $20 \%$ by the end of 2011 (varying between 28\% and 66\% for the individual bundle items). Compliance with the individual bundle items increased over time: by the end of 2011, three out of four items reached a compliance greater than $75 \%$. However, compliance with the complete bundle reached $27 \%$.

\section{Conclusion}

This is the first patient safety program implementing a SSI-bundle on a nationwide scale. The objective of $90 \%$ compliance with the complete bundle was not met; although compliance increased over time, it remained low. Likewise, registration did not exceed $20 \%$. We recommend to prolong this program, however the implementation process must be strengthened. A qualitative study is suggested to gain insight in barriers of this process.

\section{Disclosure of interest}

None declared.

\section{Author details}

${ }^{1}$ Epidemiology and Surveillance, National Institute of Public Health and the Environment, Bilthoven, The Netherlands. ${ }^{2}$ Department of Infectious Diseases, Leiden University Medical Centre, Leiden, The Netherlands.

Published: 20 June 2013

doi:10.1186/2047-2994-2-S1-056

Cite this article as: Hopmans et al:: 0056: The introduction of a surgical site infection prevention bundle on a nationwide scale. Antimicrobial Resistance and Infection Control 2013 2(Suppl 1):056. 